\title{
Utilização de experiências musicais como terapia para sintomas de náusea e vômito em quimioterapia
}

\author{
Use of musical experiences as therapy for symptoms of nausea and vomiting in chemotherapy
}

El uso de experiencias musicales como terapia para los síntomas de las náuseas y los vómitos en quimioterapia

\section{Gabriela Jorge Silva', Mirlene dos Santos Fonseca', Andrea Bezerra Rodrigues", Patrícia Peres de Oliveira"I', Débora Rabelo Magalhães Brasil", Maysa Mayran Chaves MoreiraIV}

\author{
' Hospital Israelita Albert Einstein, Faculdade de Enfermagem. São Paulo-SP, Brasil. \\ "Universidade Federal do Ceará, Centro de Ciências da Saúde, Curso de Graduação em Enfermagem. Fortaleza-CE, Brasil. \\ I"Iniversidade Federal de São João Del-Rei, Curso de Enfermagem. Divinópolis-MG, Brasil. \\ IV Instituto do Câncer do Ceará, Hospital Haroldo Juaçaba. Fortaleza-CE, Brasil.
}

Submissão: 12-12-2012 Aprovação: 26-06-2014

\section{RESUMO}

Objetivou-se aplicar as experiências musicais para avaliação dos efeitos terapêuticos em náuseas e vômitos associados à quimioterapia antineoplásica e identificar alterações nos parâmetros vitais dos pacientes que participaram da experiência. Estudo descritivo, transversal, nível II, de abordagem quantitativa, realizado com treze pacientes de um ambulatório de quimioterapia de um hospital particular no município de São Paulo. Foram utilizados dois instrumentos, sendo um deles proposto pela MASCC (Multinational Association on Supportive Care in Cancer). A maior parte da amostra foi composta por pacientes do sexo feminino, com idade entre 40 a 60 anos, casadas e com câncer de mama. Reduziu-se a frequência cardíaca em $77 \%$ da amostra; a náusea diminuiu em 100\% dos pacientes após a primeira experiência musical, e em $85 \%$ após a segunda. Concluiuse ter havido houve redução estatisticamente significativa dos sintomas de náusea e vômito após as experiências musicais.

Descritores: Quimioterapia; Musicoterapia; Náusea; Vômito; Enfermagem Oncológica.

\begin{abstract}
The study aimed to evaluate the therapeutic effects of musical experiments in nausea and vomiting associated with antineoplastic chemotherapy, and to identify changes in vital parameters of the patients who participated in the experience. This is a descriptive, transversal study, level II, which used a quantitative approach, conducted with thirteen patients from an outpatient chemotherapy unit, of a private hospital in São Paulo City. Two instruments were used, one of them proposed by MASCC (Multinational Association on Supportive Care in Cancer). The participants were predominantly females, aged 40 to 60 years, married and with breast cancer. Heart rate has decreased in $77 \%$ of the sample, and the reduction of nausea occurred in $100 \%$ of patients after the first musical experience, and in $85 \%$ after the second one. Patients reported disbelief in music in relieving nausea and vomiting before the sessions, and relief of nausea after them. It was concluded that there was a statistically significant reduction of the symptoms nausea and vomiting after the musical experiences.
\end{abstract}

Key words: Drug therapy; Music therapy; Nausea; Vomiting; Oncologic Nursing.

\section{RESUMEN}

Objetivó-se evaluar los efectos terapéuticos de experimentos musicales en las náuseas y los vómitos asociados con la quimioterapia del cáncer, e identificar cambios en los parámetros vitales de los pacientes sometidos a esa experiencia. Estudio descriptivo, exploratorio, transversal, nivel II, con enfoque cuantitativo, llevado a cabo con 13 pacientes de un ambulatorio de quimioterapia, en un hospital privado en São Paulo. En la recopilación de datos, utilizó-se dos instrumentos, uno que fue propuesto por la MASCC (Multinational Associationon Supportive Care in Cancer). Lla mayoría de los participantes estaba compuesta por pacientes de sexo femenino, con edades entre 40 y 60 años, casados y con cáncer de mama. Con respecto a los signos vitales, hubo reducción de la frecuencia cardíaca en 77\%; e de las náuseas en el $100 \%$ de los pacientes después de la primera experiencia musical, y en el $85 \%$ después de la segunda. Antes de las sesiones, los pacientes reportaron falta de fe en la música para el alivio de las náuseas y los vómitos; y alivio de las náuseas después de las sesiones. En conclusión, hubo una reducción estadísticamente significativa en las puntuaciones de náuseas y vómitos después de las experiencias musicales. Palabras clave: Quimioterapia; Musicoterapia; Náusea; Vómito; Enfermería Oncológica.

\section{AUTOR CORRESPONDENTE Maysa Mayran Chaves Moreira E-mail: maysa.mayran@gmail.com}




\section{INTRODUÇÃO}

A incidência do câncer vem crescendo a cada dia no Brasil e no mundo, e estima-se que, em 2020, o número de casos anuais seja da ordem de 15 milhões $^{(1)}$.

A quimioterapia (QT) constitui uma das modalidades de maior opção para o tratamento do câncer. De acordo com sua finalidade, a QT pode ser classificada em: curativa, que objetiva a erradicação de evidências das células cancerosas; paliativa, que visa minimizar sintomas decorrentes da proliferação tumoral aumentando a sobrevida. Pode ainda ser classificada de acordo com o período do tratamento em que é realizada, podendo ser adjuvante, ou seja, realizada depois de um tratamento principal, como por exemplo, a cirurgia e; neoadjuvante, quando é realizada previamente ao tratamento principal ${ }^{(1-2)}$.

As principais toxicidades da quimioterapia são: mielodepressão, náuseas e vômitos, alopecia, toxicidade renal, cardiotoxicidade, toxicidade pulmonar, neurotoxicidade, lesão gonadal e esterilidade, de acordo com a droga ou a dose ${ }^{(3-4)}$.

Entre os pacientes em tratamento quimioterápico, as náuseas e os vômitos estão entre os efeitos colaterais mais temidos, pois desencadeiam grande desconforto, além de prejudicarem a condição nutricional, o equilíbrio hidroeletrolítico e a qualidade de vida do indivíduo. Muitas vezes, esses efeitos tornam-se fonte de intensa ansiedade e estresse e, não raro, contribuem para o abandono do tratamento ${ }^{(3,5-7)}$.

A náusea pode ser definida como uma sensação subjetiva de incômodo gástrico, período anterior ao ato de vomitar, compreende vários outros sinais autônomos característicos como: salivação, palidez, dilatação pupilar, bradicardia ou taquicardia, variações da pressão arterial, ventilação profunda, rápida e irregular. O vômito é conceituado como a expulsão do conteúdo gástrico através da boca, geralmente é precedido por náusea e frequentemente associado com ânsia (contrações repetitivas da parede abdominal sem expulsão de

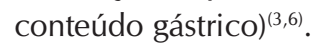

Visto o impacto negativo que as náuseas e os vômitos podem trazer aos pacientes oncológicos em QT, pretendeu-se com esse estudo aplicar as experiências musicais para avaliação dos efeitos terapêuticos desse método nos sintomas de náuseas e vômitos associados à quimioterapia antineoplásica, e identificar as mudanças ocasionadas nos parâmetros vitais (frequência cardíaca, respiratória e pressão arterial antes e após a intervenção musical terapêutica) desse grupo de pacientes.

\section{A música como uma estratégia de cuidado}

A música é um elemento que faz parte das diferentes sociedades, desde as mais antigas às atuais. Cada uma destas sociedades utiliza a música de diversas maneiras dando a ela funções específicas. Os escritos relacionados à civilização egípcia nos mostram que a música era utilizada como recurso terapêutico ${ }^{(8)}$.

Efeitos fisiológicos e psicológicos com a utilização da música são descritos no decorrer dos séculos. Durante a Idade Média, artistas menestréis tocavam para as pessoas doentes para acelerar o processo de cura. No século XVIII, Browne afirmou que o canto implica no sistema respiratório, aumenta a pressão na expiração, exercita o pulmão para os asmáticos e é prejudicial para qualquer pessoa acometida de inflamações pulmonares $^{(9)}$.

Já no século XIX, Dogiel publicou os efeitos fisiológicos gerados pela música de forma determinante; concluindo que a música altera a pressão sanguínea, os batimentos cardíacos, o sistema respiratório e o circulatório, através das alterações dos elementos musicais: altura, intensidade e timbre. Tarchanoff divulgou também em 1894 que as melodias alteram a força muscular do homem ${ }^{(9)}$.

O controle integral da náusea e vômitos pode variar para cada indivíduo, uma vez que outros fatores podem induzir ou potencializar esses efeitos colaterais, como: fatores psicológicos, comportamentais, idade, gênero, índice de massa corpórea (IMC) elevado, quadros de ansiedade e experiência prévia com QT implicando, assim, na necessidade de ações individualizadas e adaptadas para cada pessoa ${ }^{(8)}$.

Os quimioterápicos são classificados em quatro níveis de intensidade emetogênica, de acordo com a frequência dos episódios de vômito. As consideradas nível 4 são altamente emetogênicas, onde a incidência de vômitos é superior a $90 \%$. Nas consideradas de nível 3, a incidência de êmese varia de 30 a 90\%, e nas de nível 2, aquelas com baixa incidência de êmese, em torno de 30\%. Consideradas como nível 1 estão aquelas que causam muito baixa incidência de êmese (menos que $10 \%)^{(3,6)}$.

A análise de alguns estudos evidencia a associação dos saberes e práticas de utilização da música para a saúde com práticas médicas associadas às experiências musicais, trazendo efeitos fisiológicos que envolvem mudanças no metabolismo, liberação de adrenalina, regulação de frequência respiratória, variações na pressão arterial sanguínea, redução da fadiga, do tônus muscular e aumento do limiar dos estímulos sensoriais, melhorando a atenção e a concentração. Este método pode ser usado como um recurso terapêutico complementar no manejo e no controle dos sintomas, inclusive advindos do tratamento oncológico ${ }^{(10-12)}$. Sendo assim, questiona-se: as experiências musicais podem aliviar os sintomas de náuseas e vômitos associados à quimioterapia antineoplásica?

A musicoterapia é o campo da medicina que estuda o complexo som-ser humano-som, para utilizar o movimento, o som e a música com o objetivo de abrir canais de comunicação no ser humano, para produzir efeitos terapêuticos. A música e/ ou seus elementos (som, ritmo, melodia e harmonia) são utilizados, em geral, por um musicoterapeuta qualificado, com um cliente ou grupo, que objetiva desenvolver potenciais ou restabelecer funções do indivíduo para que ele possa alcançar uma melhor qualidade de vida ${ }^{(11)}$.

No contexto da Enfermagem, a música tem sido utilizada como recurso complementar no cuidado ao ser humano, em todas as idades, objetivando restaurar o equilíbrio e o bem estar possível, além de favorecer a comunicação e, muitas vezes, a ampliação da consciência individual no processo saúde/doença ${ }^{(9,11)}$.

A musicoterapia não se vale apenas da utilização da música, mas, sim, das experiências musicais do indivíduo, ou seja, não é somente a música (elemento externo ao indivíduo) que atua na terapia, mas toda forma de interação entre essa 
pessoa e a experiência que tem com a música, incluindo a interação entre pessoas, processos, produtos e contextos. Na música, existem diferentes tipos de experiências e cada um desses tipos tem seus potencias específicos e suas aplicações terapêuticas $^{(11-12)}$.

A partir desses pressupostos lançou-se a hipótese de que as experiências musicais são capazes de minimizar náuseas e vômitos apresentados por pacientes submetidos à quimioterapia antineoplásica.

O presente estudo teve como objetivo aplicar as experiências musicais para avaliação dos efeitos terapêuticos em náuseas e vômitos associados à quimioterapia antineoplásica, e identificar se ocorrem alterações nos parâmetros vitais dos pacientes que participam dessas experiências.

\section{MÉTODO}

Trata-se de um estudo descritivo, transversal, nível II, que utilizou os recursos da abordagem quantitativa. O estudo foi realizado em um ambulatório de quimioterapia de um hospital geral, particular, de grande porte, localizado no município de São Paulo. Com base em amostragem de conveniência, adotou-se como critério de seleção, pacientes oncológicos admitidos no referido ambulatório de quimioterapia que fizeram uso de quimioterápicos com moderado ou alto potencial emetogênico, no período de julho a dezembro de 2010, e que concordaram em participar da pesquisa preenchendo e assinando o Termo de Consentimento Livre e Esclarecido (TCLE). Treze pacientes participaram do estudo.

Os critérios para inclusão na pesquisa foram pacientes com idade superior a 18 anos que estavam sendo submetidos à quimioterapia antineoplásica com as seguintes drogas consideradas de alto ou moderado potencial emetogênico: carmustine, cisplatina, ciclofosfamida (com dose menor ou maior que $1500 \mathrm{mg} / \mathrm{m}^{2}$, via oral e em dias consecutivos), dacarbazina, mecloretamina, carboplatina, epirrubicina (dose inferior que $90 \mathrm{mg} / \mathrm{m}^{2}$ ), idarrubicina, ifosfamida, irinotecano, citarabina (dose maior $1000 \mathrm{mg} / \mathrm{m}^{2}$ ), dactinomicina, mitoxantrona (dose superior $12 \mathrm{mg} / \mathrm{m}^{2}$ ), daunorrubicina, oxaliplatina, doxorrubicina e procarbazina (via oral) ${ }^{(3,5)}$ e conscientes, sendo verificado o nível de consciência mediante a aplicação da Escala de Coma Glasgow, com escore igual a 15.

Para a coleta dos dados foram utilizados dois instrumentos: o primeiro, elaborado pelas autoras da pesquisa, composto por questões sobre os dados sociodemográficos, a pontuação da Escala de Coma Glasgow, a doença oncológica e as associadas, o protocolo quimioterápico, os antieméticos em uso, os hábitos sociais (consumo de álcool e/ou tabaco), e a verificação de parâmetros vitais - pressão arterial (PA), frequência cardíaca (FC) e frequência respiratória (FR), feita antes e após as sessões de musicoterapia. O outro instrumento utilizado foi um formulário elaborado pela Multinational Association of Supportive Care in Cancer (MASCC) adaptado pelas autoras para atender aos objetivos da pesquisa (Anexo A).

O estudo foi submetido e aprovado pelo Comitê de Ética em Pesquisa do Hospital Israelita Albert Einstein sob o protocolo número10/1385. Previamente à coleta, foi realizada uma solicitação formal de autorização para a coleta dos dados à responsável pelo ambulatório de quimioterapia.

Também, seguindo protocolo formal para testagem da pesquisa, foi realizado contato com a enfermeira do campo de estudo para verificar quais pacientes atendiam aos critérios de inclusão e agendamento para realização das sessões de musicoterapia.

A seguir, os pacientes oncológicos selecionados, foram orientados quanto aos objetivos do estudo e entregue o TCLE. Posteriormente, para aqueles que manifestaram o desejo de participar da pesquisa, foram verificados os sinais vitais e aplicados os instrumentos de coleta.

Para a intervenção musical terapêutica foram trabalhados os elementos fundamentais da música (melodia, harmonia e ritmo) e sua relação com a natureza humana dos pacientes (vida fisiológica, vida mental/emocional, vida mental/cognitiva e social).

Os aportes teóricos que fundamentaram a pesquisa buscaram referências terapêuticas em Edgard Willems ${ }^{(13)}$ e da musicoterapia de Benenzon ${ }^{(8)}$ e Bruscia ${ }^{(11)}$.

Ao término de cada sessão, foi realizada novamente a aferição dos sinais vitais, bem como entregue ao entrevistado o instrumento da MASCC. O participante foi orientado quanto ao preenchimento do mesmo e a trazê-lo no segundo dia de quimioterapia preenchido.

Para a comparação dos efeitos das experiências musicais nas náuseas e vômitos associados à quimioterapia antes e após as sessões foi utilizado o teste de Wilcoxon que consiste em um teste não paramétrico, equivalente ao $T$ student, e foi utilizado para comparar a diferença entre os escores de náuseas antes e após as duas experiências musicais. O teste de Wilcoxon compreende um teste usado quando os dados não atendem às suposições básicas do teste $T$ student.

\section{RESULTADOS}

\section{Caracterização da amostra}

Foram coletados dados de 13 pacientes, sendo que oito fizeram duas sessões de musicoterapia e cinco apenas uma, pois, no decorrer da pesquisa, alguns pacientes foram internados por motivos outros que não náusea e vômito.

A maior parte da população foi composta por pacientes do sexo feminino $(61 \%)$, com idade entre 40 a 60 anos $(60 \%)$, casados (77\%) e com câncer de mama (31\%).

No Quadro 1 visualizam-se os quimioterápicos utilizados em cada paciente e as medicações coadjuvantes para controle de náusea e vômito. Houve uma combinação de drogas de alto nível emetogênico, moderado e baixo.

Os pacientes $n^{\circ} 2,3,4,6,7$ e 11 utilizaram quimioterápicos de moderado risco emetogênico, representando a maioria $(46 \%)$ da amostra. Dois pacientes ( $n^{\circ} 12$ e 13 ) receberam quimioterápicos com moderado e baixo risco emetogênico concomitantemente, representando $15 \%$ amostra. Outros dois pacientes ( $n^{\circ} 8$ e 10) receberam quimioterápicos com alto potencial emetogênico $(15 \%)$. Um paciente ( $\left.n^{\circ} 9\right)$ recebeu uma combinação de drogas de alto e moderado nível emetogênico. 
Quadro 1 - Distribuição dos quimioterápicos, antieméticos e medicações adjuvantes para náusea e vômito por paciente participante do estudo. São Paulo-SP, 2010

\begin{tabular}{|c|c|c|}
\hline Paciente & Quimioterápico & $\begin{array}{l}\text { Antieméticos e medicações } \\
\text { adjuvantes para náusea/vômito }\end{array}$ \\
\hline 1 & $\begin{array}{l}\text { Doxorrubicina }-200 \mathrm{mg} / \mathrm{m}^{2} \\
\text { Cisplatina }-40 \mathrm{mg} / \mathrm{m}^{2} \\
\text { Etoposideo }-100 \mathrm{mg} / \mathrm{m}^{2} \\
\text { Mitoxantrone via oral - } 0.5 \mathrm{mg}\end{array}$ & $\begin{array}{l}\text { Dexametasona - } 8 \mathrm{mg} \\
\text { Granisentrona - } 1 \mathrm{mg} \\
\text { Lorazepam - } 0.5 \mathrm{mg}\end{array}$ \\
\hline 2 & $\begin{array}{l}\text { Doxorrubicina }-97 \mathrm{mg} / \mathrm{m}^{2} \\
\text { Ciclofosfamida }-972 \mathrm{mg} / \mathrm{m}^{2}\end{array}$ & $\begin{array}{l}\text { Aprepitante }-125 \mathrm{mg} \\
\text { Ondasetrona }-16 \mathrm{mg} \\
\text { Dexametasona }-12 \mathrm{mg} \\
\text { Aprepitante }-125 \mathrm{mg} \\
\text { Ondasetrona }-16 \mathrm{mg} \\
\text { Dexametasona }-12 \mathrm{mg}\end{array}$ \\
\hline 3 & $\begin{array}{l}\text { Irinotecano- } 85 \mathrm{mg} \\
\text { Carboplatina- } 490 \mathrm{mg}\end{array}$ & $\begin{array}{l}\text { Dexametasona - } 8 \mathrm{mg} \\
\text { Ondasetrona }-8 \mathrm{mg}\end{array}$ \\
\hline 4 & Carboplatina - 720 mg/m² & $\begin{array}{l}\text { Aprepitante }-125 \mathrm{mg} \\
\text { Dexametasona- } 20 \mathrm{mg} \\
\text { Granisentrona }-1 \mathrm{mg} \\
\text { Lorazepam- } 0.5 \mathrm{mg}\end{array}$ \\
\hline 5 & Gencitabina - 1780 mg & $\begin{array}{l}\text { Ondasetrona- } 4 \mathrm{mg} \\
\text { Dexametasona- } 4 \mathrm{mg}\end{array}$ \\
\hline 6 & $\begin{array}{l}\text { Cisplatina }-2 \mathrm{mg} / \mathrm{m}^{2} \\
+ \text { Radioterapia associada todos os dias }\end{array}$ & $\begin{array}{l}\text { Dexametasona- } 20 \mathrm{mg} \\
\text { Palonosentrona - } 0.25 \mathrm{mg}\end{array}$ \\
\hline 7 & $\begin{array}{l}\text { Epirrubicina - } 170 \mathrm{mg} \\
\text { Ciclofosfamida }-865 \mathrm{mg}\end{array}$ & $\begin{array}{l}\text { Aprepitante- } 125 \mathrm{mg} \\
\text { Dexametasona - 1mg } \\
\text { Granisentrona - 1mg }\end{array}$ \\
\hline 8 & Cisplatina - $78 \mathrm{mg}$ & $\begin{array}{l}\text { Granisentrona - } 1 \mathrm{mg} \\
\text { Dexametasona - } 15 \mathrm{mg}\end{array}$ \\
\hline 9 & $\begin{array}{l}\text { Doxorrubicina }-57,5 \mathrm{mg} \\
\text { Dacarbazina }-860 \mathrm{mg}\end{array}$ & $\begin{array}{l}\text { Ondasetrona - } 4 \mathrm{mg} \\
\text { Dexametasona - } 10 \mathrm{mg}\end{array}$ \\
\hline 10 & Cisplatina - 30 mg/m² & $\begin{array}{l}\text { Granisentrona - } 1 \mathrm{mg} \\
\text { Dexametasona - } 20 \mathrm{mg} \\
\text { Lorazepam - 0,5 mg }\end{array}$ \\
\hline 11 & $\begin{array}{l}\text { Ciclofosfamida - } 965 \mathrm{mg} \\
\text { Doxorrubicina - } 95 \mathrm{mg}\end{array}$ & $\begin{array}{l}\text { Dexametasona - } 12 \mathrm{mg} \\
\text { Granisentrona }-1 \mathrm{mg} \\
\text { Aprepitante }-125 \mathrm{mg}\end{array}$ \\
\hline 12 & $\begin{array}{l}\text { Ciclofosfamida }-600 \mathrm{mg} \\
\text { Metrotexate }-40 \mathrm{mg} / \mathrm{m}^{2}\end{array}$ & $\begin{array}{l}\text { Dexametasona - } 20 \mathrm{mg} \\
\text { Palonosentrona - 0,25 mg }\end{array}$ \\
\hline 13 & $\begin{array}{l}\text { Carboplatina - } 167 \mathrm{mg} \\
\text { Paclitaxel - } 158 \mathrm{mg} \\
\text { Gencitabina - } 1580 \mathrm{mg}\end{array}$ & $\begin{array}{l}\text { Granisentrona - } 1 \mathrm{mg} \\
\text { Dexametasona - } 10 \mathrm{mg}\end{array}$ \\
\hline
\end{tabular}

Fonte: elaboração das autoras.
Todos os pacientes estavam fazendo uso de, pelo menos, uma droga quimioterápica de alto ou moderado potencial emetogênico, com exceção do paciente $n^{\circ} 5$ que estava em tratamento com gencitabina, droga classificada de baixo potencial emetogênico. No entanto, esse paciente foi incluído, pois apresentava queixa de êmese constante e foi indicado pela nutricionista do ambulatório para a sessão de musicoterapia.

Os pacientes fizeram uso de antieméticos antes da infusão do quimioterápico, conforme os guidelines propostos internacionalmente pela National Comprehensive Cancer Network (NCCN) e pela American Society of Clinical Oncology (ASCO), inclusive o fármaco aprepitante, medicação inibidora de neuroquinina (NK1), preconizada para protocolos quimioterápicos de moderado e alto potencial emetogênico.

Com relação aos sinais vitais, não houve alterações significativas na pressão arterial da maioria dos pacientes. Contudo, houve redução da frequência cardíaca em $77 \%$ da amostra (pacientes $n^{\circ} 1,2,4,5,6,8$, $10,11,12,13)$; e redução da frequência respiratória na maioria dos pacientes, com exceção do paciente $\mathrm{n}^{\circ}$ 7, após as sessões de musicoterapia.

Visualiza-se na Tabela 1 que não houve significância estatística na variação dos sinais vitais antes e após as sessões de musicoterapia, utilizando-se o teste $T$ de Student, onde o p foi sempre maior que 0,05 .

Tabela 1 - Correlação entre as médias de sinais vitais antes e após as sessões de musicoterapia. São Paulo-SP, 2010

\begin{tabular}{|c|c|c|c|c|}
\hline Variável & $\mathbf{N}$ & Média & Desvio padrão & Valor $\mathbf{p}<0,05$ \\
\hline PAs ${ }^{1}$ antes da primeira experiência musical & 21 & 118,10 & 14,36 & NS \\
\hline PAs após a primeira experiência musical & 21 & 118,57 & 13,15 & NS \\
\hline PAd $^{2}$ antes da primeira experiência musical & 21 & 73,81 & 6,69 & NS \\
\hline PAd após a primeira experiência musical & 21 & 74,29 & 7,46 & NS \\
\hline $\mathrm{FC}^{3}$ antes da segunda experiência musical & 21 & 80,81 & 12,44 & NS \\
\hline FC após a segunda experiência musical & 21 & 78,24 & 10,82 & NS \\
\hline $\mathrm{FR}^{4}$ antes da segunda experiência musical & 21 & 18,286 & 1,488 & NS \\
\hline FR após a segunda experiência musical & 21 & 18,095 & 1,338 & NS \\
\hline
\end{tabular}

Fonte: Dados do estudo.

${ }^{1} \mathrm{PAs}=$ pressão arterial sistólica; ${ }^{2} \mathrm{PAd}=$ pressão arterial diastólica; ${ }^{3} \mathrm{FC}=$ frequência cardíaca; ${ }^{4} \mathrm{FR}=$ frequência respiratória; $N S=$ não significativo 
Tabela 2 - Comparação entre as médias de náuseas antes e após as experiências musicais pelo Teste de Wilcoxon. São Paulo-SP, 2010

\begin{tabular}{lcccc}
\hline Variável & $\mathbf{n}$ & Média & $\begin{array}{l}\text { Desvio } \\
\text { padrão }\end{array}$ & $\begin{array}{l}\text { Valor } \mathbf{p} \\
<0,05\end{array}$ \\
\hline $\begin{array}{l}\text { Náuseas antes da } \\
\text { primeira experiência } \\
\text { musical }\end{array}$ & 13 & 1,923 & 1,441 & \\
$\begin{array}{l}\text { Náuseas após a } \\
\text { primeira experiência } \\
\text { musical }\end{array}$ & 13 & 0,000000 & 0,000000 & \\
$\begin{array}{l}\text { Wilcoxon Test } \\
\text { Náuseas antes da } \\
\text { segunda experiência } \\
\text { musical }\end{array}$ & 09 & 4,78 & 3,63 & \\
$\begin{array}{l}\text { Náuseas após a } \\
\text { segunda experiência } \\
\text { musical }\end{array}$ & 09 & 0,556 & $\mathbf{0 , 0 0 1}$ & \\
Wilcoxon Test & & & & \\
\hline
\end{tabular}

Fonte: Dados do estudo.

Observa-se na Tabela 2 que houve redução significativa de náuseas antes e após as sessões de musicoterapia. Antes e após a primeira sessão houve significância no Teste de Wilcoxon de 0,001, e antes e após a segunda experiência musical, a significância foi de 0,005.

\section{DISCUSSÃO}

O presente estudo teve como objetivo aplicar as experiências musicais para avaliação dos efeitos terapêuticos em náuseas e vômitos associados à quimioterapia antineoplásica e identificar alterações nos parâmetros vitais.

Todos os pacientes na presente pesquisa utilizavam antieméticos de primeira geração como antagonistas de 5HT3 (ondasentrona, granisentrona, entre outros), e de segunda geração (palonosentrona e antagonistas de NK1 - aprepitante) que permitem um ótimo controle de náusea e vômito causados pela quimioterapia $^{(2)}$. No entanto, o número de pacientes que apresentavam náuseas ou vômitos na primeira experiência musical sugere que, somente a terapia farmacológica, não controla totalmente os sintomas em $100 \%$ dos casos de pacientes submetidos à quimioterapia de alto ou moderado potencial emetogênico.

Em grande ensaio clínico randomizado, cerca de 50\% dos doentes em tratamento com quimioterapia de elevado risco emético tiveram vômitos, e 58\% experienciaram náuseas, apesar de submetidos à terapia antiemética ${ }^{(14)}$.

Neste estudo, foi possível observar que não houve alterações estatisticamente significativas com relação aos parâmetros vitais. Em um estudo sobre os efeitos terapêuticos da música após cirurgia cardíaca em 79 crianças, em uma Unidade de Terapia Intensiva, foi verificado uma contribuição importante da música no controle da dor, da ansiedade e na redução dos sinais vitais, principalmente na frequência cardíaca e respiratória ${ }^{(15)}$.
Já com relação ao escore de náusea e vômito houve redução estatisticamente significativa, assim como em outro estudo que utilizou também técnica não farmacológica para controle desses sintomas, evidenciando que apenas a abordagem farmacológica não é suficiente para o controle total dos mesmos em pacientes submetidos à quimioterapia de alto $\mathrm{e}$ moderado potencial emético ${ }^{(16)}$, confirmando a hipótese dos autores de que tais efeitos poderiam ser reduzidos com as experiências musicais. Pesquisa realizada nos Estados Unidos da América afirmou que a música reduziu significativamente a incidência de náuseas e vômitos induzida por quimioterapia, e que pode ser usada como um abordagem de apoio no processo de quimioterapia para o paciente ${ }^{(17)}$.

Em uma reflexão teórica sobre a importância das intervenções não farmacológicas direcionadas a procedimentos de enfermagem para pacientes com câncer, discute-se e enfatiza-se a musicoterapia como recurso relevante na área de enfermagem $^{(18)}$. Assim, torna-se necessária a inserção de programas de educação permanente em serviço de saúde com treinamento para o uso de terapias complementares, a fim de aumentar o interesse e a sensibilidade das equipes de profissionais que trabaIham nessa área ${ }^{(19-20)}$, especialmente em setores de oncologia.

Destarte, os estudos supracitados reforçam e corroboram os achados da presente pesquisa, que mostrou que o uso de técnica não farmacológica é uma alternativa para o controle de náuseas e vômitos. O presente estudo, dentro das limitações da amostra, demonstrou que a musicoterapia foi uma importante ferramenta para minimizar a náusea e vômito associados à quimioterapia antineoplásica, visto que houve uma diferença estatística significativa $(p<0,005)$ na percepção dos participantes da pesquisa depois das experiências musicais.

Pode-se verificar que a musicoterapia vem sendo utilizada para diferentes tipos de população e com diferentes objetivos, como redução de ansiedade e dor. No entanto, houve dificuldade na obtenção de estudos sobre a utilização desta terapia em pacientes oncológicos com náusea e vômito.

Ressalta-se que, para se obter uma intervenção musical eficiente, foi considerado neste estudo, aspectos importantes de acordo com os aportes teóricos ${ }^{(8,11,13)}$ como a preferência musical, o tempo de intervenção, os atributos e natureza da música, o desejo do indivíduo em participar de atividades envolvendo música, a idade e os efeitos fisiológicos desencadeados pela música.

Diversos autores orientam sobre a estratégia de se considerar a preferência musical do paciente para a eficácia da terapia $^{(8,11-12,15-17)}$, pois o êxito do tratamento complementar depende do prazer despertado pela música, fazendo com que o cérebro da pessoa privilegie sensações prazerosas em detrimento dos efeitos do tratamento quimioterápico.

Em relação à natureza e aos atributos da música, como o ritmo, a harmonia, a melodia e o volume, esses precisam ser levados em consideração de acordo com os efeitos que se almeja alcançar com a intervenção musical ${ }^{(11,16-17)}$. Músicas com ritmo e melodia mais calmas e com tons mais graves são as mais indicadas quando se deseja proporcionar sensações de tranquilidade, pois estes componentes podem reduzir a agitação, a ansiedade e promover relaxamento e prazer ${ }^{(8,12)}$. 
Segundo Lee e colaboradores, os profissionais acreditam nos efeitos benéficos da musicoterapia ${ }^{(18)}$. Esse talvez seja o primeiro passo no sentido de aplicação dessa terapia, dentre outras possíveis, nas diferentes áreas ${ }^{(18)}$ de saúde. Acredita-se que muitos profissionais da saúde não utilizam essa forma de terapia, provavelmente, por não se sentirem preparados para tal.

Destarte, deve-se frisar que a intervenção musical traz benefícios tanto fisiológicos quanto psicológicos para pessoas em qualquer faixa etária( ${ }^{(8,11,17)}$ e pode-se constituir em um recurso eficaz para qualificar o cuidado ao paciente oncológico, além de ser uma intervenção de baixo custo, não farmacológica e não invasiva.

O enfermeiro pode ser um facilitador do processo do uso da musicoterapia, expandindo sua atuação através da implantação da intervenção musical nos serviços de saúde, participando não só como executor do projeto, mas também da avaliação de sua eficácia. Assim, possibilitará a amenização do sofrimento de pacientes, especialmente os oncológicos em tratamento quimioterápico. Mas, para isso, o profissional precisará buscar conhecimentos específicos para saber como atuar, avaliar e orientar os pacientes e demais membros da equipe de saúde sobre os benefícios dessa terapia complementar.

\section{CONCLUSÃO}

Embora a música tenha influenciado minimamente nas modificações dos parâmetros vitais, houve redução estatisticamente significativa de náuseas e vômitos antes e após as experiências musicais. Assim, apesar de não permitir generalizações, em razão do número de pacientes estudados, pode-se concluir que a utilização da música diminuiu e proporcionou alívio nas náuseas e vômitos dos participantes.

Ressalta-se que o estudo apresenta como limitação o reduzido tamanho amostral, decorrente de ter sido realizado em uma única unidade ambulatorial com os pacientes submetidos a quimioterapia antineoplásica em uso de drogas consideradas de alto ou moderado potencial emetogênico, apesar de estar de acordo com os critérios estipulados no método. Contudo, essa limitação serve como orientação para estudos futuros mais aprofundados que ratifiquem os resultados positivos e estimulem a continuidade desse tipo de avaliação com grupos maiores de pacientes e em diferentes espaços hospitalares para uma possível confirmação desses resultados preliminares.

Os dados apresentados sinalizam para a utilização da musicoterapia como abordagem terapêutica adjuvante no tratamento quimioterápico para portadores de câncer, pois contribui para a redução de náusea e vômito e, consequentemente, para a melhoria da qualidade de vida do indivíduo em uso de drogas antineoplásicas.

Pretende-se que os resultados deste estudo incentivem o uso de terapias complementares na prática da Enfermagem. Entretanto, é necessário que o profissional esteja motivado, seja qualificado e treinado para realizar as intervenções com segurança, de acordo com os princípios éticos, respeitando as preferências do ser humano e apropriando-se do domínio da terapia a ser utilizada.

\section{REFERÊNCIAS}

1. Ministério da Saúde (BR), Instituto Nacional do Câncer (BR). Ações de enfermagem para controle do câncer: uma proposta de integração ensino-serviço. 3. ed. Rio de Janeiro (RJ): INCA; 2008.

2. Johnston PG, Spence RAJ. Oncologia. Rio de Janeiro (RJ): Guanabara Koogan; 2003.

3. Bonassa EMA, Gato MIR. Terapêutica oncológica para enfermeiros e farmacêuticos. 4. ed. São Paulo (SP): Atheneu; 2011.

4. Ballatori E, Roila F. Impact of nausea and vomiting on quality of life in cancer patients during chemotherapy. Health Qual Life Outcomes [Internet]. 2003 [cited 2012 december 12];1:46. Available from: http://www.ncbi.nlm. nih.gov/pubmed/14521717

5. Bergkvist K, Wengstrom Y. Symptom experiences during chemotherapy treatment-with focus on nausea and vomiting. Eur J Oncol Nurs [Internet]. 2006 [cited 2012 december 12];10:21-9. Available from: http://www.ncbi.nlm. nih.gov/pubmed/15908274

6. Soriano García JL, Lima Pérez M, González González J, Barbán Suárez R, García Diéguez R, Batista Albuerne N. Náuseas y vómitos inducidos por quimioterapia antineoplásica. Rev Cuba Med. 2006;45(2):112-22.
7. Giglio A, Mota A. Novos avanços no controle da náusea pós quimioterapia antineoplásica. Rev Bras Mastologia. 1998;8(4):196-203.

8. Benenzon RO. Musicoterapia: de la teoría a la práctica. Buenos Aires (AR): Paidós; 2000.

9. Taets GGC, Barcellos LRM. Música no cotidiano de cuidar: um recurso terapêutico para enfermagem. Rev Pesqui Cuid Fundam. 2010;2(3):1009-16.

10. Santos M, Pinho M, Silva S, Dias V. Estudo sobre emese aguda e tardia em doentes a efectuar quimioterapia, alta e moderadamente emetizantes em internamento. Onco News [Internet]. [acesso em 12 de dezembro de 2012]. Disponível em: www.aeop.net/sgc/.../DOCS/bc4c2b62176e8d9818fa088d886747f2.pdf

11. Bruscia KE. Definindo musicoterapia. 2. ed. Rio de Janeiro (RJ): Enelivros; 2000.

12. Karagozoglu S, Tekyasar F, Yilmaz FA. Effects of music therapy and guided visual imagery on chemotherapy-induced anxiety and nausea-vomiting. J Clin Nurs [Internet]. 2012 [cited 2012 december 12 ];22(1-2):39-50. Available from: http://www.ncbi.nlm.nih.gov/pubmed/23134272

13. Willems E. Las bases psicológicas de la educación musical. Buenos Aires: Eudeba; 1961. 
14. Waar DG. Chemotherapy and cancer related nausea and vomiting. Curr Oncol [Internet]. 2008 [cited 2012 december 12];15(1):1-9. Available from: http://www.ncbi. nlm.nih.gov/pmc/articles/PMC2216421/

15. Hatem TP, Lira PI, Mattos SS. The therapeutic effects of music in children following cardiac surgery. J Pediatr [Internet]. 2006 [cited 2012 december 12];82(3):186-92. Available from: http://www.ncbi.nlm.nih.gov/pubmed/16680285

16. Mandel SE, Hanser SB, Ryan LJ. Effects of a music-assisted relaxation and imagery compact disc recording on health-related outcomes in cardiac rehabilitation. Music Ther Perspect [Internet]. 2010 [cited 2012 december 12];28(1):11-21. Available from: http://mtp.oxfordjournals.org/content/28/1/11.short?rss $=1 \&$ ssource $=\mathrm{mfr}$

17. Gimeno MM. The effect of music and imagery to induce relaxation and reduce nausea and emesis in patients with cancer undergoing chemotherapy treatment.
Music Medicine [Internet]. 2010 [cited 2012 december 12];2(3):174-81. Available from: http://mmd.sagepub. com/content/2/3/174.abstract

18. Lee YY, Chan MF, Mok E. Effectiveness of music intervention on the quality of life of older people. J Adv Nurs [Internet]. 2010 [cited 2012 december 12];66(12):2677-87. Available from: http://www.ncbi.nlm.nih.gov/pubmed/20831571

19. Caminha LB, Silva MJP, Leão ER. The influence of musical rhythms on the perception of subjetive states of adult patients on dialysis. Rev Esc Enferm USP [Internet]. 2009 [cited 2012 december 12];43(4):923-9. Available from: http://www.ncbi.nlm.nih.gov/pubmed/20085165

20. Burns JL, Labbé E, Arke B, Capeless K, Cooksey B, Steadman $A$, et al. The effects of different types of music on perceived and physiological measures of stress. J Music Ther [Internet]. 2002 [cited 2012 december 12];39(2):101-16. Available from: http://www.ncbi.nlm.nih.gov/pubmed/12213081

\section{ANEXO A - FORMULÁRIO ADAPTADO DE INSTRUMENTO ANTIEMÉTICO MASCC (MULTINATIONAL ASSOCIATION ON SUPORTIVE CARE IN CANCER)}

Náusea e Vômito antes e durante as primeiras 24 horas após a quimioterapia:

Náusea e Vômito antes e durante as primeiras 24 horas após a quimioterapia:

1) Você está apresentando náusea neste momento?

Sim ( ) Não ( )

2) Nas últimas 24 horas você teve náuseas?

Sim ( ) Não ( )

3) Você vomitou nas últimas 24 horas?

Sim ( ) Não ( )

Fonte: MASCC-Multinational Association of Supportive Care in Cancer, 2004. Adaptado pelas autoras da pesquisa.

Por favor, preencha este quadro no dia seguinte ao dia da Quimioterapia.

Náusea e Vômito nas 24 horas após a quimioterapia:

1) Nas últimas 24 horas você teve náuseas: Sim ( ) Não ( )

Quantas vezes?

De zero (nenhuma náusea) a dez (náusea insuportável) quanto você daria de nota para a náusea que apresentou?

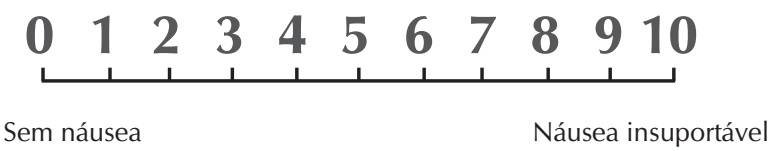

2) Você vomitou nas últimas 24 horas: $\quad \operatorname{Sim}($ ) Não ( )

Quantas vezes? 